\title{
PERIODIC SOLUTIONS OF SYSTEMS OF DIFFERENTIAL EQUATIONS ${ }^{1}$
}

\author{
IRVING J. EPSTEIN
}

Introduction and summary. Let $A$ be an $n \times n$ matrix whose elements are continuous functions of the real variable $t$. Consider the system

$$
Y=A Y \quad\left(\cdot=\frac{d}{d t}\right)
$$

where $Y$ is the unknown $n \times n$ matrix. We show that, without loss of generality, $A$ may be assumed to be either symmetric $\left(A^{\prime}=A\right.$, $A^{\prime}=$ transpose of $\left.A\right)$ or skew symmetric $\left(A^{\prime}=-A\right)$, since (1) can be replaced by two systems in which one matrix is symmetric and the other skew symmetric.

We shall be concerned with the question of periodic solutions of a particular system of the type

$$
Y=\Sigma Y
$$

where $\Sigma$ is skew symmetric and periodic with period $\omega$. If $\Sigma$ is also odd, $(\Sigma(t)=-\Sigma(-t))$, Demidovic [1] has shown that $Y$ will be periodic. We give below a simple proof of this result, in which moreover the hypothesis that the matrix be skew symmetric is dropped. If $\Sigma$ is not odd, nothing beyond the Floquet theory is known about the periodicity of $Y$.

When $\Sigma$ is a $3 \times 3$ matrix we shall use a theorem in differential geometry, which is due to Fenchel [2], to obtain a necessary condition on the elements of $\Sigma$, in order that $Y$ be periodic with period $\omega$.

Results and proofs. We shall denote the unit matrix by $I$. We have:

THEOREM 1. The solution of $\dot{Y}=A Y, Y(0)=I$ with $A$ arbitrary is determined when we have the solutions of

$$
\dot{\mathcal{\Theta}}=\Sigma \cdot \Theta ; \quad \Theta(0)=I, \quad \Sigma=\frac{1}{2}\left(A-A^{\prime}\right)
$$

and

Received by the editors May 24, 1961 and, in revised form, September 29, 1961.

' I acknowledge with pleasure the advice given me by Professor W. Magnus of New York University in the preparation of this paper. 


$$
\dot{Z}=S \cdot Z ; \quad Z(0)=I, \quad S=\mathcal{O}^{\prime}\left(\frac{A+A^{\prime}}{2}\right) \vartheta
$$

and is in fact $Y=O Z$. (We note that $\Sigma$ is skew symmetric and that $S$ is symmetric and depends on $\theta$, the solution of $\dot{\theta}=\Sigma \theta$.)

Proof. The proof follows from a simple calculation and from the obvious fact that $\theta$ must be orthogonal.

Demidovic has shown that if $\Sigma$ is skew symmetric, odd $(\Sigma(t)$ $=-\Sigma(-t))$ and periodic with period $\omega$, then all solutions of $\dot{Y}=\Sigma Y$ are periodic with either period $\omega$ or $2 \omega$. We give below a simpler proof of this result, dropping the hypothesis of skew symmetry. In addition we prove all solutions have period $\omega$.

THEOREM 2. If $\Sigma$ is odd and periodic with period $\omega$ then all solutions of $\dot{Z}=\Sigma Z$ are periodic with period $\omega$.

Proof. The solution $\dot{Z}=\Sigma Z ; Z(0)=I$ is unique. Let $Z(-t)=H(t)$. We have

$$
\frac{d H}{d t}=-\dot{Z}(-t)=-\Sigma(-t) Z(-t)=\Sigma(t) H(t) .
$$

Since $Z(t)$ and $H(t)$ satisfy the same equation and since moreover $Z(0)=H(0)=I$ we have by the uniqueness of the solution that $Z(-t)=H(t)=Z(t)$. If we consider now the matrix $Z(t+\omega)$ we see that it satisfies our differential equation equation and we obtain $Z(t+\omega)=Z(t) F$ where $F$ is a constant matrix (Floquet's theory). If we now let $t=-\omega / 2$ in the last equation we get $F=I$, since $Z(-t)$ $=Z(t)$ and $Z(t)$ is nonsingular. Hence $Z(t+\omega)=Z(t)$ which completes the proof.

We return now to the equation $\dot{Y}=A Y ; Y(0)=I$ in which we restrict $A$ to be a $3 \times 3$ skew symmetric matrix which in addition is continuous and periodic with real period $\omega$.

$$
A(t)=\left(\begin{array}{ccc}
0 & a(t) & b(t) \\
-a(t) & 0 & c(t) \\
-b(t) & -c(t) & 0
\end{array}\right)
$$

where $a(t), b(t), c(t)$ are real periodic functions of $t$ with common period $\omega$. We have:

Lemma 1. Let $A$ be given by (2). Consider the system $\dot{Y}=A Y$. There exists a constant orthogonal matrix $O$ such that, in the skew symmetric matrix

$$
\mathcal{O}^{\prime} A \Theta=\tilde{A}
$$


the element a corresponding to $a(t)$ in $A$ satisfies the relation

$$
\mu(\tilde{a}(t))=0
$$

where, for any function $f(t)$,

$$
\mu(f)=\frac{1}{\omega} \int_{0}^{\omega} f(t) d t .
$$

Proof. If $\mu(a)=0$, take $\theta=I$. Otherwise, choose $\theta$ so that its third column is orthogonal to the vector with the components,

$$
\mu(c), \quad-\mu(b), \quad \mu(a) .
$$

The rest follows from a straightforward computation.

If we introduce $\tilde{Y}=O Y$ as the unknown matrix, it satisfies the differential equation

$$
\dot{\bar{Y}}=\tilde{A} \tilde{Y}
$$

Therefore, we may assume that $a(t)$ in (1) has already the property $\mu(a)=0$, since $\tilde{Y}$ will have periodic elements if and only if the same is true for $Y$.

Lemma 1 provides the possibility for reduction of the system $\dot{Y}=A Y$. We have:

Lemma 2. If the matrix $A(t)$ defined by (1) has the property that $A(t+\omega)=A(t)$ and $\mu(a)=0$, then the system

$$
\dot{Y}=A Y, \quad Y(0)=I
$$

has a periodic solution $Y$ of period $\omega$ if and only if the system

$$
\dot{U}=B U, \quad U(0)=I
$$

has a periodic solution $U$ of period $\omega$, where

$$
B(t)=\left(\begin{array}{rcc}
0, & 0, & \beta \\
0, & 0, & \gamma \\
-\beta, & -\gamma, & 0
\end{array}\right)
$$

and

$$
\begin{aligned}
& \beta(t)=b \cos \alpha-c \sin \alpha, \\
& \gamma(t)=b \sin \alpha+c \cos \alpha, \\
& \alpha(t)=\int_{0}^{t} a(s) d s .
\end{aligned}
$$

Obviously, $B(t+\omega)=B(t)$ since 


$$
\alpha(t+\omega)=\alpha(t)+\mu(a)=\alpha(t) .
$$

Proof. Let $\theta$ be defined by

$$
\theta=\left(\begin{array}{ccc}
\cos \alpha & \sin \alpha & 0 \\
-\sin \alpha & \cos \alpha & 0 \\
0 & 0 & 1
\end{array}\right)
$$

and put

$$
Y=\theta U
$$

Then $\dot{Y}=A Y$ implies

$$
U=\theta^{-1}(A \theta-\dot{\theta}) U
$$

and a simple calculation shows that (10) is identical with (3).

Obviously, the system (3) with the matrix (4) is nothing but the Frenet-Serret formulas of differential geometry with curvature $\beta$ and torsion $\gamma$. In obtaining information about the periodicity of $U$ we shall use certain results from differential geometry.

If $U$ is periodic with period $\omega$ then regarding $t$ as arc length we see that from $Z$ to $Z+\omega$ along a curve in 3 space the curvature, torsion, tangent, normal and binormal are periodic. We now consider the spherical indicatrix of the tangents to this curve. For this purpose we assume that $\beta(t)>0$ and that $\beta(t)$ and $\gamma(t)$ are differentiable. A calculation shows us that the curvature $\beta_{1}$ and torsion $\gamma_{1}$ of our spherical indicatrix are given respectively by

$$
\beta_{1}^{2}=\frac{\beta^{2}+\gamma^{2}}{\beta^{2}} \quad \text { and } \quad \gamma_{1}=\frac{\beta \gamma^{\prime}-\beta^{\prime} \gamma}{\beta\left(\beta^{2}+\gamma^{2}\right)} \quad\left(\prime^{\prime}=\frac{d}{d t}\right)
$$

and that the arc lengths are connected by $d t_{1} / d t=\beta(t)$ where $t_{1}$ is the arc length of the spherical indicatrix. Now there is a result due to Fenchel [2] concerning the integral curvature of a curve which states that the integral curvature, $\int|\kappa| d s$, of a closed space curve is equal to or greater than $2 \pi$. Here $s$ is the arc length of the curve and the limits of integration are the length of the curve. If our $U$ is to be periodic the spherical indicatrix of the tangents is a closed curve. Hence we have:

A necessary condition that $U$ given by $\dot{U}=B U$, where $B$ is given by (4) and where $\beta$ and $\gamma$ are real, differentiable, periodic functions of $t$ with period $\omega$ and where $\beta(t)>0$, be periodic is that $\int_{0}^{\infty}\left(\beta^{2}+\gamma^{2}\right)^{1 / 2} d t$ $\geqq 2 \pi$.

It should be remarked that we do not get additional inequalities if we consider the spherical indicatrix of the normals or binormals or 
for that matter if we consider the spherical indicatrices of the spherical indicatrices.

We close by stating the above results in terms of the matrix $A$ given by equation (2).

THEOREM 3. A necessary condition that the solution of $\dot{Y}=A Y$, $Y(0)=I$, should be periodic with period $\omega$ where $A$ is given by (2) and where $a(t), b(t), c(t)$ be periodic with period $\omega$ and satisfy

$$
b \cos \int_{0}^{t} a(\xi) d \xi-c \sin \int_{0}^{t} a(\xi) d \xi>0
$$

is that

$$
\int_{0}^{\omega}\left(b^{2}+c^{2}\right)^{1 / 2} d t \geqq 2 \pi
$$

\section{REFERENCES}

1. B. P. Demidovic, Moskov. Gos. Univ. Ux. Zap. 6 (1952), 123-132.

2. W. Fenchel, Math. Ann. 101 (1929), 238-252.

U. S. Army Signal Research and Development Laboratory, Fort Monmouth, NeW Jersey 